\title{
CCR4 is a prognostic biomarker and correlated with immune infiltrates in head and neck squamous cell carcinoma
}

\author{
Yijian Zhang ${ }^{1 \#}$, Kai Chen ${ }^{2 \#}$, Li Li ${ }^{3 \#}$, Weidong $\mathrm{Mao}^{4}$, Dong Shen ${ }^{4}$, Ninghua $\mathrm{Yao}^{5}$, Lei Zhang ${ }^{1}$ \\ ${ }^{1}$ Department of Otolaryngology-Head and Neck Surgery, Affiliated Hospital of Jiangnan University, Wuxi, China; ${ }^{2}$ Department of Radiotherapy, \\ First People's Hospital of Yancheng, Yancheng, China; ${ }^{3}$ Department of Oncology, Huaian Hospital, Huai'an, China; ${ }^{4}$ Department of Oncology, \\ Jiangyin People's Hospital, Wuxi, China; ${ }^{5}$ Department of Oncology, Affiliated Hospital of Nantong University, Nantong, China \\ Contributions: (I) Conception and design: Y Zhang, L Zhang; (II) Administrative support: L Zhang, W Mao; (III) Provision of study materials or \\ patients: K Chen; (IV) Collection and assembly of data: L Li; (V) Data analysis and interpretation: Y Zhang, D Shen; (VI) Manuscript writing: All \\ authors; (VII) Final approval of manuscript: All authors. \\ \#These authors contributed equally to this work. \\ Correspondence to: Lei Zhang. Department of Otolaryngology-Head and Neck Surgery, Affiliated Hospital of Jiangnan University, Wuxi, China. \\ Email: wxzhl@139.com.
}

Background: Increased evidence has indicated that the tumour microenvironment plays an essential in the development, treatment and prognosis of head and neck squamous cell carcinoma (HNSC). Recent studies have indicated CC chemokine receptor 4 (CCR4) plays an essential role in tumor invasion and other adverse biological behavior. This study used data from the Cancer Genome Atlas (TCGA) database to explore the role of CCR4 in HNSC and its clinical significance.

Methods: The gene expression and clinical data of HNSC patients in the TCGA database were extracted. Gene Expression Profiling Interactive Analysis (GEPIA) was used to analyze the expression of CCR4 in tumor and non-tumor tissue. Kaplan-Meier survival analysis was used to analyze the relationship between CCR4 expression and overall survival rate (OS), disease-specific survival (DSS), and progression-free interval (PFI) in HNSC. A logistic regression model was used to analyze the relationships between various clinical factors and CCR4 expression. Gene Set Enrichment Analysis (GSEA) was used to explore the potential role of CCR4 in HNSC. Additionally, we explored the relationship between CCR4 and immune infiltration.

Results: The expression of CCR4 in HNSC was not significantly different from that in normal tissue. The expression level of CCR4 in wild-type TP53 was higher than that in mutant TP53. Cox regression analysis showed the expression level of CCR4 was related to the patient's tumor grade and Tumor-Node-Metastasis (TNM) stage. CCR4 expression level is an independent prognostic factor. CCR4 is positively correlated with immune infiltration and immune checkpoints expression levels. The results of GSEA revealed that the high CCR4 expression group genes were enriched in allograft rejection, inflammatory response, IL-6/JAK/ STAT3 signaling, interferon gamma response, and KRAS signaling up. Low CCR4 expression group genes were enriched in oxidative phosphorylation, MYC targets v1, DNA repair, reactive oxygen species pathway, and P53 pathway. Further, our study indicated CCR4 can also predict the prognosis of radiotherapy patients. Conclusions: Our study found that CCR4 was a prognostic marker related to HNSC immune infiltration, and patients with high expression of CCR4 had a better prognosis.

Keywords: CC chemokine receptor 4 (CCR4); head and neck squamous cell carcinoma (HNSC); biomarker; immune infiltrates

Submitted Jul 16, 2021. Accepted for publication Sep 02, 2021.

doi: 10.21037/atm-21-3936

View this article at: https://dx.doi.org/10.21037/atm-21-3936

\footnotetext{
^ ORCID: 0000-0002-7607-2516.
} 


\section{Introduction}

Head and neck tumors refer to tumors located above the clavicle and below the skull base. About $90 \%$ of head and neck malignant tumors are squamous cell carcinoma (1). Head and neck squamous cell carcinoma (HNSC) has a high rate of recurrence and distant metastasis, creating a significant health burden worldwide (2). Early diagnosis and treatment can result in a better prognosis. Common risk factors for HNSC include tobacco, alcohol, environmental risk, and human papillomavirus (HPV) (3). HPV-negative tumors correlate with a worse prognosis (4). No reliable biomarkers have been defined beyond HPV.

Considerable progress has been made in the diagnosis and treatment of HNSC, and surgical treatment, radiotherapy, and chemotherapy can significantly improve the disease control rate and survival rate of patients with HNSC. However, the overall survival rate of patients is still relatively low, with a 5 -year survival rate at all stages of about $40-50 \%$ (1,5-7). Although immunotherapy has improved the survival rate of some patients in recent years, effective methods to judge the prognosis of patients are still lacking. It is therefore important to find effective prognostic markers.

As a large group of structurally-related cytokines, chemokines play an important role in migration, homing, and tumor microenvironment $(8,9)$. There is still controversy over the link between chemokines and prognosis in different types of cancers because chemokines have both pro- and anticancer properties (10). CC chemokine receptor $4(C C R 4)$ is a receptor of $\mathrm{CC}$ chemokine ligand 22 (CCL22) and CC chemokine ligand 17 (CCL17) and can affect the function of $\mathrm{T}$ helper type 2 (Th2) and regulatory $\mathrm{T}$ cells (Tregs).

CCR4 is expressed on the surface of T lymphocytes, NK cells, monocytes, macrophages, and eosinophils (11). In T lymphocytes, CCR 4 is mainly expressed on the surface of Th2 cells and CD4+, CD25+ and Treg. It has different functions, so CCR4 can be divided into: CCR4+ Th2 cells and CCR4+ Treg cells (12). CCR4 is expressed on the surface of Treg cells as one of the characteristics of Treg cells. Lechner et al. (13) confirmed that compared with normal tissues, Treg cells levels are significantly higher in HNSC and have systemic immunosuppressive activity. When the chemokine TARC/MDC secreted by tumor cells and tumor-infiltrating macrophages binds to the ligand through the receptor CCR4, the Treg cells at the tumor site are transported, resulting in a large number of Treg cells aggregation and infiltration in the tumor microenvironment, making the composition of effector cells and immune regulatory cells in tumor infiltrating lymphocytes (TILs) has changed, which further affects the biological behavior of tumors (12).

Studies have shown that CCR4 is highly expressed in different tumors and in tumor invasion, and that it plays an essential role in other adverse biological behaviors (14-16). Because CCR4 recruits cancer-related cells into the tumor microenvironment, patients with high expression of CCR4 or its chemokines seemed to have a poor prognosis in renal cancer and testicular cancer. In contrast, high expression of CCR 4 or its chemokines improves the prognosis in lung cancer and melanoma patients $(15,17,18)$. There are few reports on the expression of CCR 4 in HNSC tissue and whether CCR4 is related to tumor prognosis. Although studies have shown that CCR4 is essential for building a microenvironment that stimulates the anti-tumor immune response to inhibit tumor growth and migration, it has not been evaluated whether CCR 4 can be used as an independent prognostic factor (19). This study analyzed the relationship between CCR4 and tumor immune infiltration level and clinical tumor prognosis to provide support for further research on HNSC treatment strategies. We present the following article in accordance with the REMARK reporting checklist (available at https://dx.doi.org/10.21037/ atm-21-3936).

\section{Methods}

\section{The Cancer Genome Atlas (TCGA) data acquisition}

Level 3 RNA-sequencing data (Fragments Per Kilobase per Million, FPKM) for 33 types of human cancer were download from TCGA through the National Institute of Cancer's Genomic Data Commons (GDC) data portal (https://portal.gdc.cancer.gov/). RNA-sequencing data for HNSC and corresponding clinical information were also downloaded from TCGA. RNA sequencing data were transformed to transcripts per million reads (TPM) and normalized into $\log 2(\mathrm{TPM}+1)$. HNSC baseline characteristics are detailed in Table $\mathrm{S} 1$ in the appendix. The study was conducted in accordance with the Declaration of Helsinki (as revised in 2013).

\section{Tumor Immune Estimation Resource (TIMER) database analysis}

The TIMER database (https://cistrome.shinyapps.io/ 
timer/) was used to analyze the correlation between CCR4 expression level and immune cell infiltration. TIMER was also used to conduct survival analysis for immune cells and CCR4 in HNSC.

\section{Gene Set Enrichment Analysis (GSEA)}

GSEA (http://software.broadinstitute.org/gsea/index.jsp) was conducted between high- and low-CCR4 expression groups to identify CCR4-related functional significance in HNSC based on hallmark gene sets ("h.all.v7.0.symbols. gmt").

\section{Additional bioinformatic and statistical analysis}

All analyses were performed with $\mathrm{R}$ version 3.6.1. To explore the expression of CCR4, Mann-Whitney nonparametric analysis was used to test unpaired differences between groups and Wilcoxon signed-rank test for paired differences. Log rank (Mantel-Cox) test was used for survival analysis. Univariable Cox proportional hazard models were applied to evaluate factors associated with overall survival. To identify independent prognostic factors, all significant variables on univariate Cox regression analysis $(\mathrm{P} \leq 0.05)$ were subjected to multivariate Cox regression analysis. Wilcox test was used for comparisons between 2 groups and the Kruskal-Wallis test was used for multigroup comparisons. Correlation analysis was performed with Spearman's correlation test. Receiver operating characteristic curves (ROCs) were plotted and areas under the curve (AUC) were calculated using the pROC package in R. Survival analysis was carried out using the R package "Survival". Univariate Cox and multivariate Cox regression analysis was carried out using the R package "glment'. Infiltration levels for different immune cell types were quantified using single-sample gene set enrichment analysis (ssGSEA) with the R package "gsva". Visualization was performed using the R package "ggplot2" (http://ggplot2. org). $\mathrm{P}<0.05$ was considered as significant.

\section{Results}

\section{Pan-cancer analysis of CCR4 expression}

The clinical data of 33 different types of tumors were downloaded from TCGA (normal $=730$, tumor $=11,363$ ). As shown in Figure 1A, CCR4 was upregulated in 17 human cancers, including breast invasive carcinoma (BRCA), cervical squamous cell carcinoma and endocervical adenocarcinoma (CESC), colon adenocarcinoma (COAD), lymphoid neoplasm diffuse large B-cell lymphoma (DLBC), esophageal carcinoma (ESCA), glioblastoma multiforme (GBM), kidney renal clear cell carcinoma (KIRC), acute myeloid leukemia (LAML), brain lower grade glioma (LGG), ovarian serous cystadenocarcinoma (OV), pancreatic adenocarcinoma (PAAD), prostate adenocarcinoma (PRAD), rectum adenocarcinoma (READ), skin cutaneous melanoma (SKCM), stomach adenocarcinoma (STAD), thyroid carcinoma (THCA), and thymoma (THYM). CCR4 was downregulated in adrenocortical carcinoma (ACC), kidney chromophobe (KICH), lung squamous cell carcinoma (LUSC), testicular germ cell tumors (TGCT), and uterine carcinosarcoma (UCS), and there was no significant change in bladder urothelial carcinoma (BLCA), cholangiocarcinoma (CHOL), HNSC, kidney renal papillary cell carcinoma (KIRP), liver hepatocellular carcinoma (LIHC), lung adenocarcinoma (LUAD), pheochromocytoma and paraganglioma (PCPG), or uterine corpus endometrial carcinoma (UCEC) compared with corresponding normal tissue. Next, the Gene Expression Profiling Interactive Analysis (GEPIA) database was used to analyze the expression profile of CCR4 in tumor samples and normal tissue. The study found that, compared with normal tissue, the expression of CCR4 in LUSC is decreased. In short, CCR4 is upregulated in BRCA, LUSC, $\mathrm{STAD}$, and $\mathrm{KIRC} / \mathrm{KICH}$, indicating that CCR4 may play a key regulatory role in the carcinogenesis of these 4 cancers.

As shown in Figure 1A, the expression of CCR4 did not cause any significant changes in HNSC compared with normal tissue. We then investigated the correlation between expression of CCR4 and survival in HNSC patients, including overall survival (OS), disease-specific survival (DSS), and progression-free interval (PFI). With respect to OS, DFS, and PFI, patients with higher expression of $C C R 4$ had a better prognosis, indicating that CCR 4 could be used as a biomarker to predict prognosis in HNSC patients (Figure 1).

\section{Survival analysis of differential expression of CCR 4 and different clinical characteristics}

In order to explore the relationship between CCR4 and various clinical characteristics, we divided the entire cohort into several subgroups to estimate survival curves for high and low expression of CCR4. As shown in Figure 2, we found that in both early- and late-stage HNSC, patients with high 


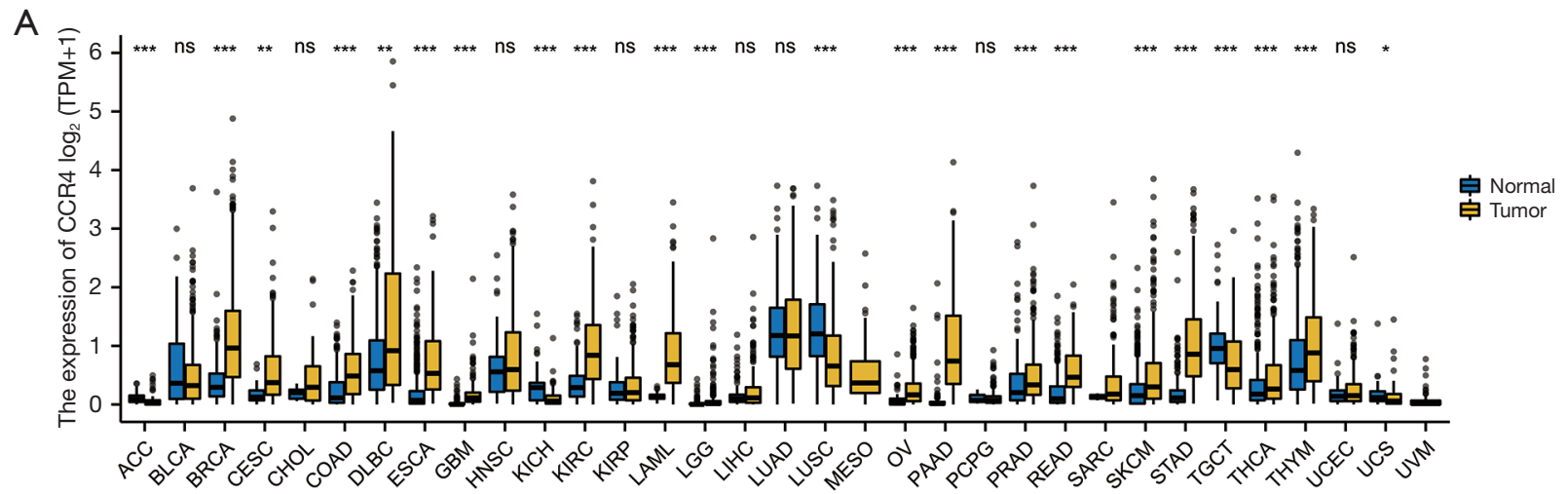

B


Figure 1 Expression analysis for CCR4 in 33 cancers and the relationship between CCR4 expression and HNSC survival prognosis. (A) CCR4 expression in 33 human cancers based on TCGA cancer data and normal data. (B) CCR4 expression in TCGA BLCA, BRCA, CHOL, COAD, ESCA, HNSC, KICH, KIRC, KIRP, LIHC, LUAD, LUSC, PAAD, PRAD, READ, STAD, THCA, and UCEC tissue compared with paired normal tissue. (C) OS of CCR4 expression in HNSC. (D) DSS of CCR4 expression in HNSC. (E) PFI of CCR4 expression in HNSC. ${ }^{*} \mathrm{P} \leq 0.05 ;{ }^{*} \mathrm{P} \leq 0.01 ;{ }^{* * *} \mathrm{P} \leq 0.001$; ns, $\mathrm{P}>0.05$. CCR4, CC chemokine receptor 4; HNSC, head and neck squamous cell carcinoma; TGCA, the Cancer Genome Atlas; BLCA, bladder urothelial carcinoma; BRCA, breast invasive carcinoma; CHOL, cholangiocarcinoma; COAD, colon adenocarcinoma; ESCA, esophageal carcinoma; KICH, kidney chromophobe; KIRC, kidney renal clear cell carcinoma; KIRP, kidney renal papillary cell carcinoma; LIHC, liver hepatocellular carcinoma; LUAD, lung adenocarcinoma; LUSC, lung squamous cell carcinoma; PAAD, pancreatic adenocarcinoma; PRAD, prostate adenocarcinoma; READ, rectum adenocarcinoma; STAD, stomach adenocarcinoma; THCA, thyroid carcinoma; UCEC, uterine corpus endometrial carcinoma; OS, overall survival; DSS, disease specific survival; PFI, progression-free interval. 
A



B



C

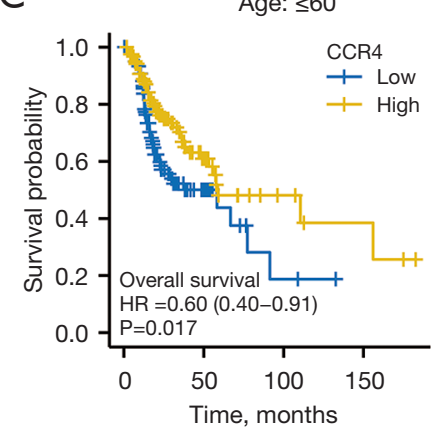

D

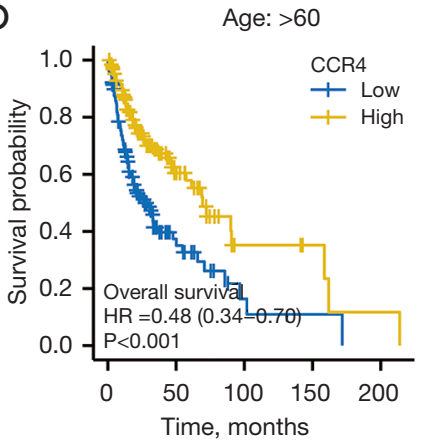

$\mathrm{E}$

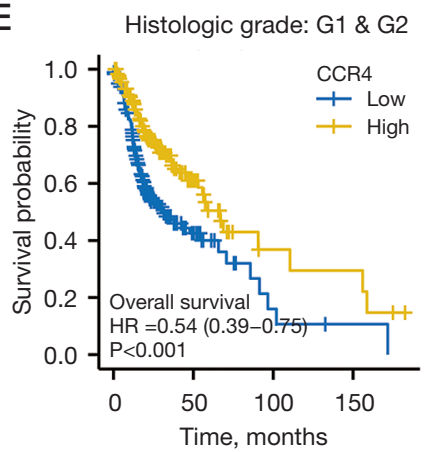

$\mathrm{F}$

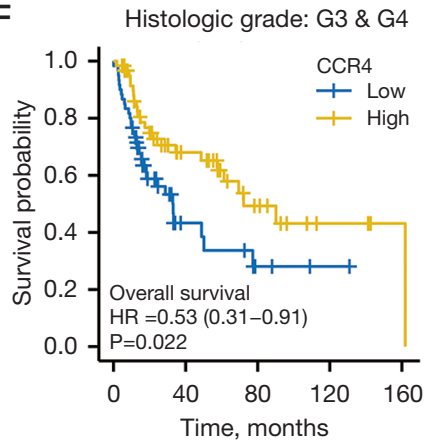

Figure 2 Survival analysis of different CCR4 subgroups. (A) Survival curve of differential expression of CCR4 in patients with T1/T2 stage HNSC; (B) survival curve of differential expression of CCR4 in patients with T3/T4 stage HNSC; (C) survival curve of differential expression of CCR4 in patients under 60 years of age; (D) survival curve of differential expression of CCR4 in patients over 60 years of age; (E) survival curve of differential expression of CCR4 in patients with G1/G2 grade; (F) survival curve of differential expression of CCR4 in patients with G3/G4 grade. CCR4, CC chemokine receptor 4; HNSC, head and neck squamous cell carcinoma.

expression of CCR4 had a better prognosis (Figure 2A,2B), and this effect was seen in patients both over and under 60 years old (Figure 2C,2D). Compared to the low CCR4 expression group, patients in the high CCR4 expression group with both low and high histologic grades showed a better prognosis (Figure $2 E, 2 F$ ). This result further shows that regardless of tumor stage I/II or III/IV, age over or under 60 years, and histologic grade G $1 / 2$ or $3 / 4$, HNSC patients with high expression of CCR 4 had a better prognosis, once again indicating that CCR4 could be used as a biomarker to predict prognosis in HNSC patients.

\section{Relationship between CCR4 expression and TP53 in HNSC}

TP53 plays an important role in HNSC as a suppressor gene. Therefore, we further explored the relationship between CCR 4 expression, prognosis, and TP53. The study cohort data included information on the presence of tumors with TP53 mutations in HNSC. TP53 mutations were present in 340 patients (68.8\%) and wild-type TP53 in 154 patients (31.2\%). As shown in Figure 3, CCR4 expression was significantly correlated with the presence of TP53 mutations. CCR4 expression of wild-type TP53 was higher and prognosis better compared to that of mutant TP 53 $(\mathrm{P}<0.05)$. In addition, we observed that the differential expression of TP53 affected the expression level of CD274. High expression of wild-type TP53 had the highest expression of CD274, and high-expressed wild-type TP53 had the longest median survival time $(\mathrm{P}<0.05)$.

\section{Relationship between CCR4 expression and clinical characteristics in HNSC}

We used Cox regression analysis to perform univariate and multivariate correlation analysis. As shown in Figure 4, tumor grade, pathological Tumor-Node-Metastasis (pTNM) stage, and CCR4 expression were all significantly correlated with overall survival. Therefore, tumor grade, 

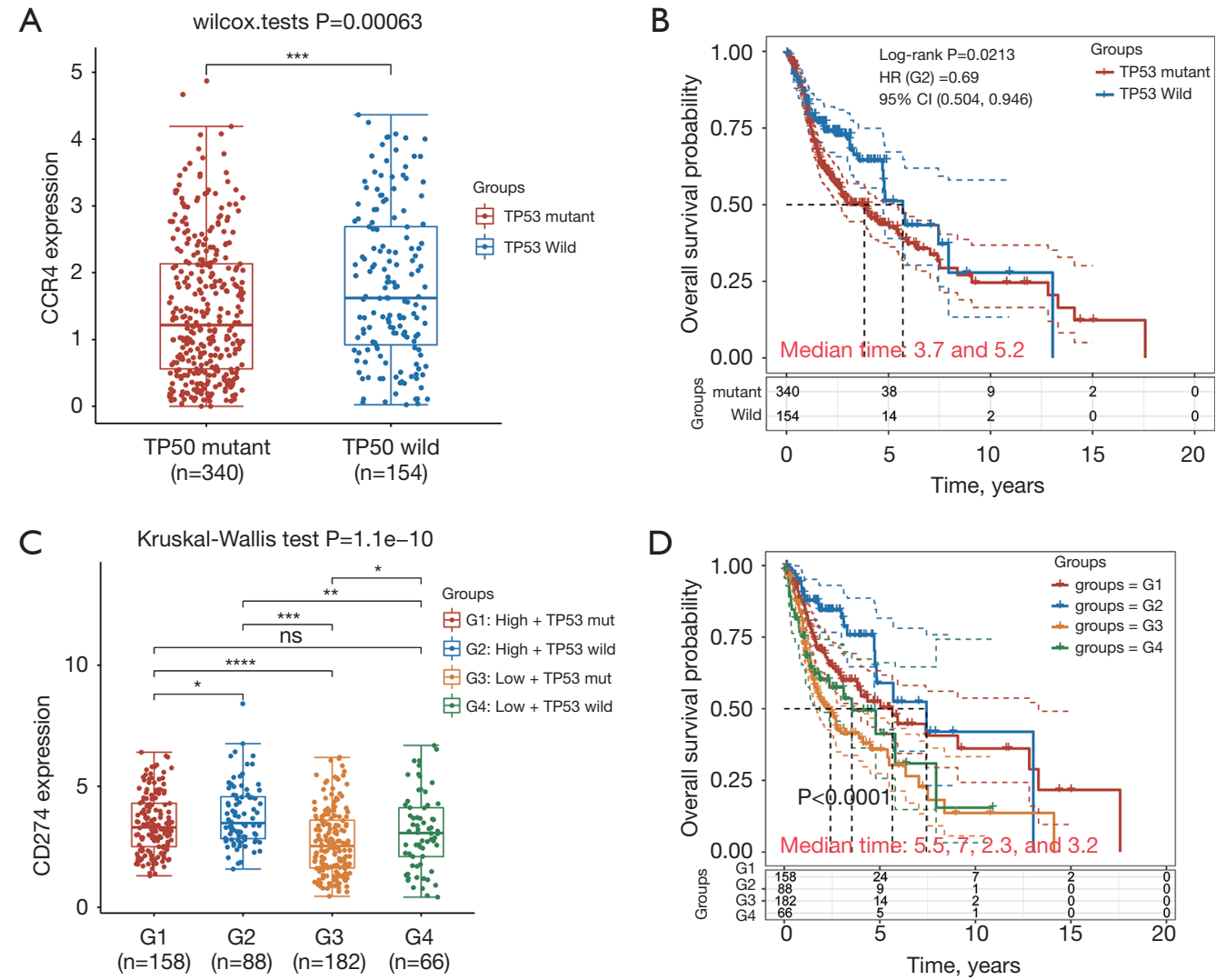

Figure 3 Expression of CCR4 in the mutant and wild-type TP53 groups and relationship with prognosis. (A) The expression of CCR4 in the wild-type TP53 group was significantly higher than that in the mutant group; (B) the prognosis of the wild-type TP53 group was better than that of the mutant TP53 group; (C) the expression of CD274 in differentially expressed wild-type and mutant TP53 groups; (D) compared to the mutant TP53 group, high expression of wild-type TP53 suggested a better prognosis. ${ }^{*} \mathrm{P} \leq 0.05 ;{ }^{* *} \mathrm{P} \leq 0.01 ;{ }^{* * *} \mathrm{P} \leq 0.001 ; \mathrm{ns}, \mathrm{P}>0.05$. CCR4, CC chemokine receptor 4 .

pTNM stage, and CCR4 expression could be considered independent prognostic factors.

\section{The relationship between CCR4 expression and tumor-infiltrating immune cells}

In the treatment of malignant tumors, tumor-infiltrating immune cells play a vital role in tumor control and treatment response. Many studies have confirmed that tumor-infiltrating immune cells are closely related to survival and prognosis in various cancers. Therefore, we investigated the relationship between CCR4 expression and immune infiltration in HNSC. The CIBERSORT analysis tool (https://cibersort.stanford.edu) was used to estimate the composition of 24 immune cells and evaluate their different concentrations in the high and low CCR4 expression groups. Figure 5 shows the proportion of immune cell subsets in differentially expressed CCR4. The high CCR4 expression group was rich in activated dendritic cells (aDC), B cells, T cells, cytotoxic cells, DC, eosinophils, immature DC (iDC), macrophages, mast cells, neutrophils, CD56 $6^{\text {dim }}$ natural killer (NK) cells, NK cells, plasmacytoid DC (pDC), T helper (Th) cells, central memory T (TCM) cells, effector memory $\mathrm{T}$ (TEM) cells, follicular helper $\mathrm{T}$ (TFH) cells, $\mathrm{T}$ helper 1 (Th1) cells, Th17 cells, Th2 cells, and Tregs $(\mathrm{P}<0.05)$. These immune cells were positively correlated with CCR 4 expression. While the tumor growth delay (TGD) was negatively correlated with CCR4 expression, the low CCR4 expression group had a higher TGD $(\mathrm{P}<0.05)$.

\section{CCR4 expression is correlated with immune infiltration level in HNSC}

Tumor infiltrating lymphocytes independently predict 


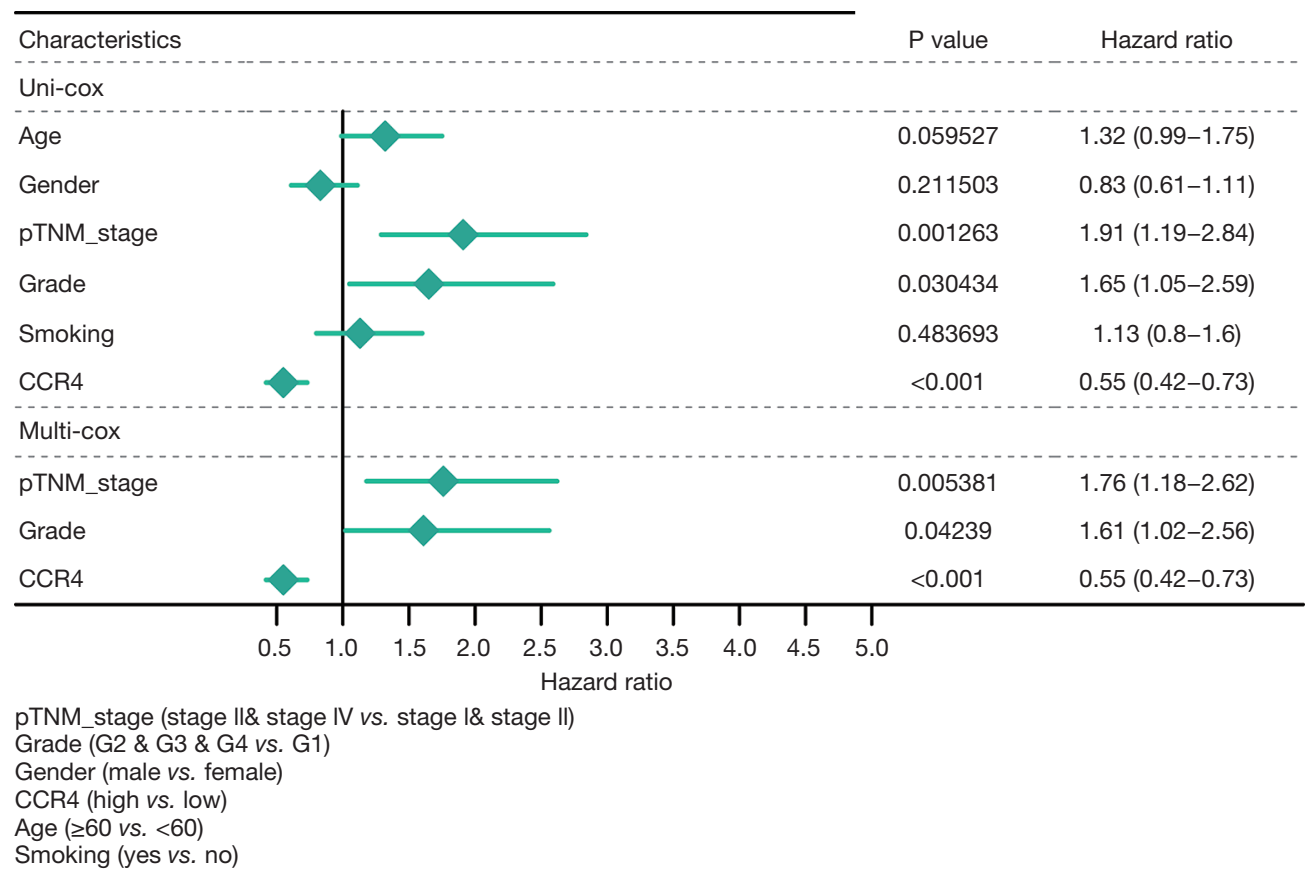

Figure 4 Association between overall survival and clinical pathological characteristics in HNSC patients using Cox regression model (with pTNM stage, tumor grade, and CCR4 expression as independent prognostic factors). CCR4, CC chemokine receptor 4; HNSC, head and neck squamous cell carcinoma.

the overall survival rate and sentinel lymph node status of cancer patients. This allowed us to use TIMER to evaluate the correlation between CCR 4 expression and the level of immune infiltration in different types of tumors. As shown in Figure 6, the expression level of CCR4 is positively correlated with B cell $(\mathrm{r}=0.484, \mathrm{P}=2.93 \mathrm{e}-29), \mathrm{CD} 4+\mathrm{T}$ cell $(\mathrm{r}=0.683, \mathrm{P}=4.20 \mathrm{e}-67), \mathrm{CD} 8+\mathrm{T}$ cell $(\mathrm{r}=0.525, \mathrm{P}=6.67 \mathrm{e}-35)$, macrophage $(\mathrm{r}=0.541, \mathrm{P}=4.39 \mathrm{e}-38)$, neutrophil $(\mathrm{r}=0.526$, $\mathrm{P}=1.75 \mathrm{e}-35)$, and $\mathrm{DC}(\mathrm{r}=0.701, \mathrm{P}=1.82 \mathrm{e}-72)$ immune infiltration level, and $\mathrm{B}$ cells, T cells, and CCR 4 are related to the cumulative survival of HNSC over time rate-related factors. The above results indicate that CCR 4 played a specific role in the level of tumor cell immune infiltration in HNSC.

\section{CCR4 expression is correlated with immune checkpoints in HNSC}

As immune checkpoints have been used in clinical treatment, we plotted the relationship between immune checkpoints and CCR4 to further clarify the relationship between CCR4 and immunity, including sialic acid binding Ig-like lectin 15 (SIGLEC15), CD274, programmed cell death 1 ligand 2 (PDCD1LG2), hepatitis A virus cellular receptor 2 (HAVCR2), cytotoxic T-lymphocyte associated protein 4 (CTLA4), lymphocyte activating 3 (LAG3), programmed cell death 1 (PDCD1), and T cell immunoreceptor with Ig and ITIM domains (TIGIT) (Figure 7A). Our results found that the expression of CCR4 was positively correlated with these 8 immune checkpoints (Figure 7B-7I). These results confirmed that the expression of CCR 4 is specifically related to immune infiltrating cells in HNSC, and that CCR4 played an important role in tumor immune escape.

\section{Gene sets enriched in CCR4 expression phenotype}

GSEA results showed that 5 Kyoto Encyclopedia of Genes and Genomes (KEGG) pathways were enriched in the high CCR4 expression group, including allograft rejection, inflammatory response, IL-6/JAK/STAT3 signaling, interferon gamma response, and KRAS signaling up (Figure $8 A$ ). Five KEGG pathways were enriched in the low CCR4 expression group, including oxidative phosphorylation, MYC targets $\mathrm{v}$, DNA repair, reactive oxygen species pathway, and P53 pathway (Figure 8B). 

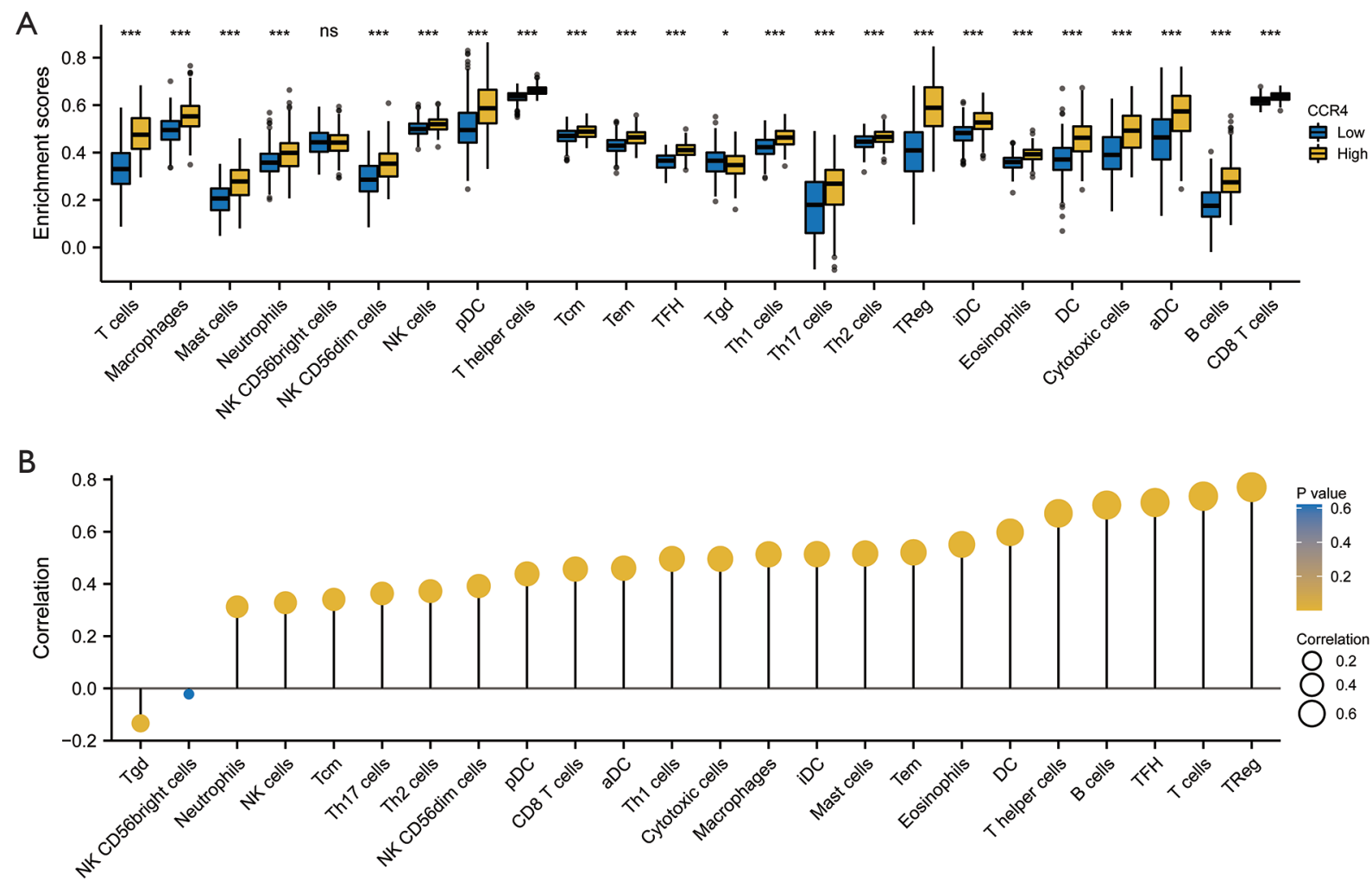

Figure 5 Infiltrated immune cells in differentially expressed CCR4 groups. (A) Difference of 24 infiltrated immune cells in CCR4 between high and low expression groups; (B) correlation between high and low expression of CCR4 and infiltrated immune cells. * $\mathrm{P} \leq 0.05$; *** $\mathrm{P} \leq 0.001$; NS: $\mathrm{P}>0.05$. CCR4, CC chemokine receptor 4.

These findings indicated that CCR4 had potential value in the development of HNSC.

\section{The prognostic value of CCR4 in patients with radiotherapy}

We evaluated the prognostic value of CCR4 in patients receiving radiotherapy (Figure 9). As shown in Figure 9A, we found that patients with high expression of CCR4 had better OS than patients with low CCR4 expression. As shown in Figure 9B, patients with high expression of CCR4 also had better PFS than patients with low CCR4 expression. After follow-up, we also found that the patients who had received radiotherapy had better OS and PFS during the 5 -year follow-up (Figure 9C,9D). These results indicated that CCR4 was also a good prognostic biomarker in HNSC patients who have received radiotherapy.

\section{Discussion}

Cellular immunity plays an important role in antitumor immunity. T cells, NK cells, and macrophages are the main effector cells of the immune system. Chemokines actively participate in the tumor immune process by affecting the distribution and function of immune cells in the body. Changes in the expression level of chemokines in the tumor microenvironment will inevitably change the number of immune cells infiltrating the tumor tissue.

Chemokines are usually divided into 4 groups consisting of $\mathrm{C}, \mathrm{CC}, \mathrm{CXC}$, and CX3C, based on conserved cysteines $(20,21)$. Most chemokine receptors recognize more than 1 chemokine. CCR4, as the receptor of the CC family, can recognize CCL17 and CCL22. CCR4 is expressed in $\mathrm{T}$ cells and plays multiple roles simultaneously, including regulating biological functions of $\mathrm{T}$ cells and Th2 cells $(22,23)$. Previous studies have reported that CCR4 regulated the traffic of dendritic cells, recirculated $T$ cells from tissue to draining lymph nodes, and migrated $\mathrm{T}$ cells to ectopic lymphoid tissue $(24,25)$. CCR4 has also been found to be expressed in some epithelial cells, such as lung, colon, and bronchial epithelial cells $(26,27)$ and to play an important 

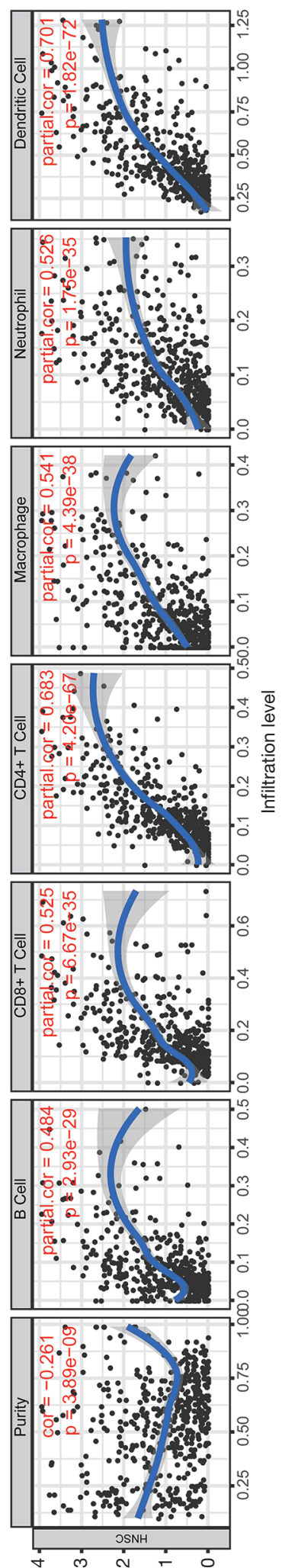

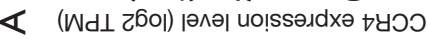

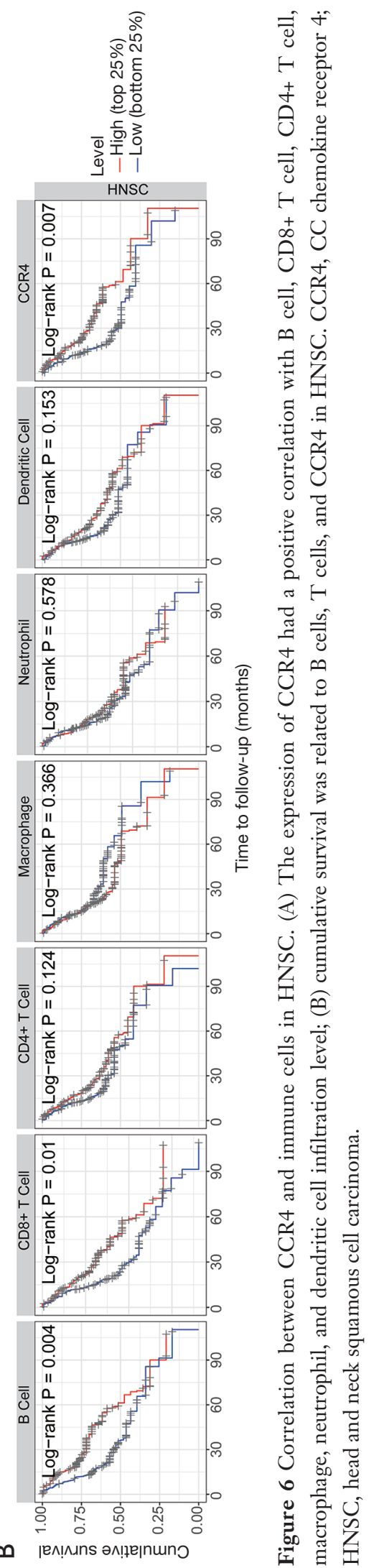


A
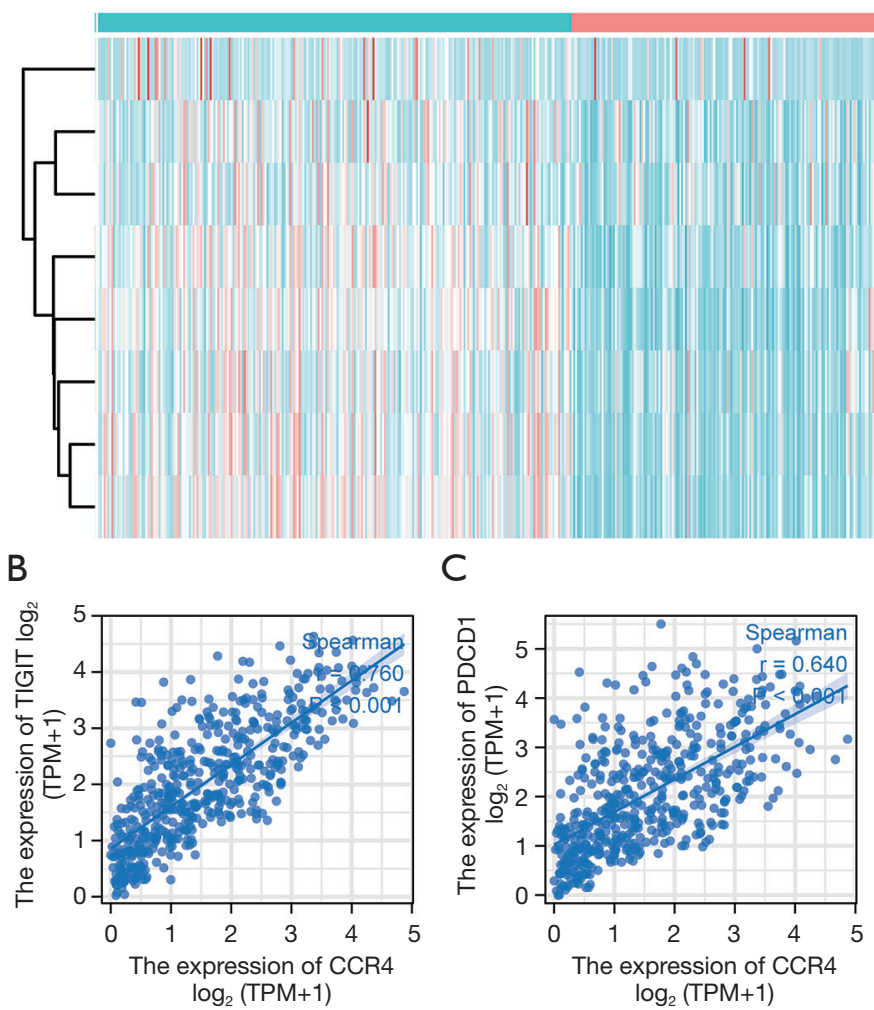

$\log _{2}(\mathrm{TPM}+1)$


Figure 7 Relationship between the expression of CCR4 and eight immune checkpoints in HNSC. (A) The relationship between CCR4 and immune checkpoints; (B) The expression correlation of CCR4 with TIGHT in HNSC; (C) the expression correlation of CCR4 with PDCD1 in HNSC; (D) the expression correlation of SIGLEC15 with CCR4 in HNSC; (E) the expression correlation of CCR4 with PDCD1LG2 in HNSC; (F) the expression correlation of CCR4 with LAG3 in HNSC; (G) the expression correlation of CCR4 with HAVCR2 in HNSC; (H) the expression correlation of CCR4 with CD274 in HNSC; (I) the expression correlation of CCR4 with CTLA4 in HNSC. ${ }^{* * *} \mathrm{P} \leq 0.001$. CCR4, CC chemokine receptor 4; HNSC, head and neck squamous cell carcinoma; HAVCR2, hepatitis A virus cellular receptor 2; SIGLEC15, sialic acid binding Ig-like lectin 15; PDCD1LG2, programmed cell death 1 ligand 2; LAG3, lymphocyte activating 3; CTLA4, cytotoxic T-lymphocyte associated protein 4.

role in many malignant tumors, including solid and hematological tumors (28-30).

Recent studies have further confirmed that chemokines are also involved in angiogenesis, embryonic development, tumor metastasis and other processes (31). Human immune cells such as T lymphocytes, NK cells, monocytes and eosinophils all have CCR4 expressed on the cell surface (32). $\mathrm{T}$ lymphocytes play a key role in the body's immune 
A

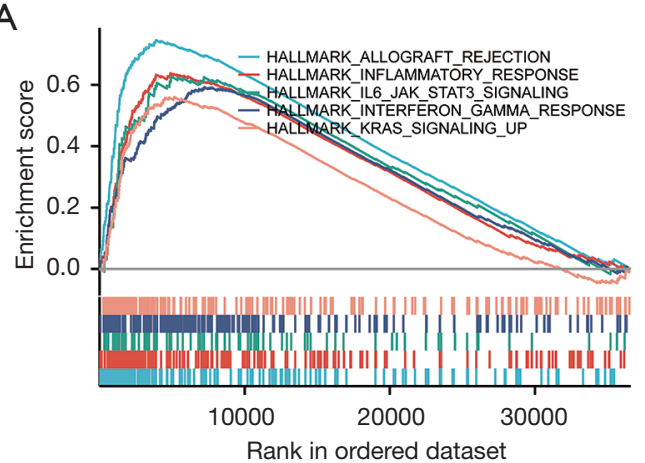

B

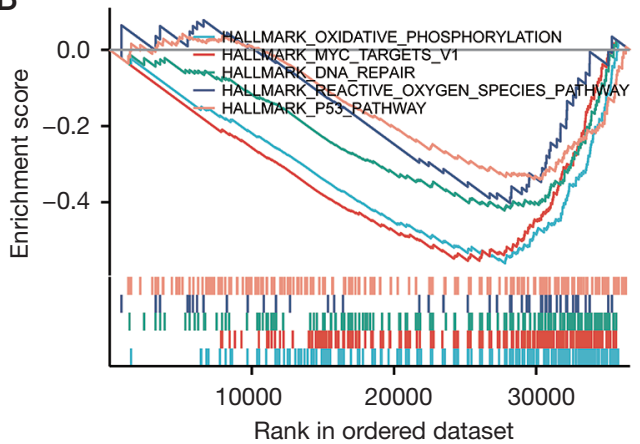

Figure 8 Enrichment plot from Gene Set Enrichment Analysis (GSEA). (A) GSEA results show that hallmark genes with high CC chemokine receptor 4 (CCR4) expression are differentially enriched; (B) GSEA results show the differential enrichment of hallmark genes with low expression of CCR4. GSEA, Gene Set Enrichment Analysis; CCR4, CC chemokine receptor 4.
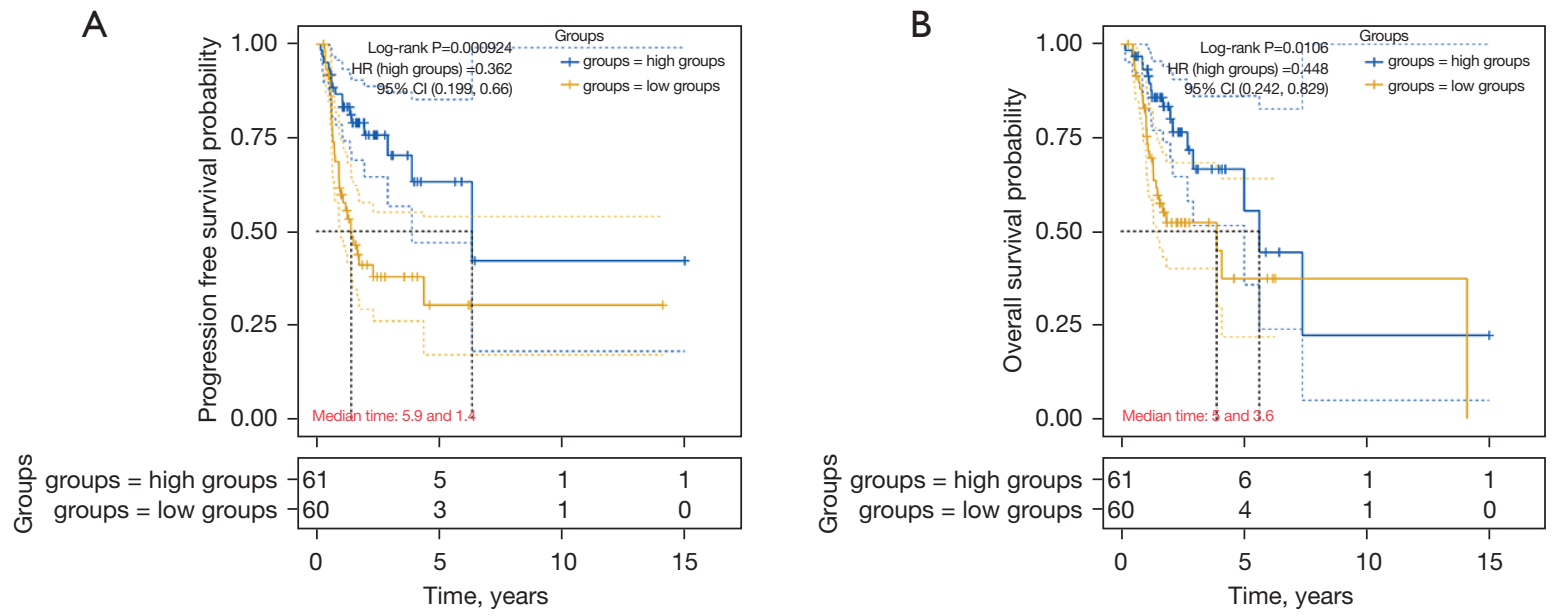

C
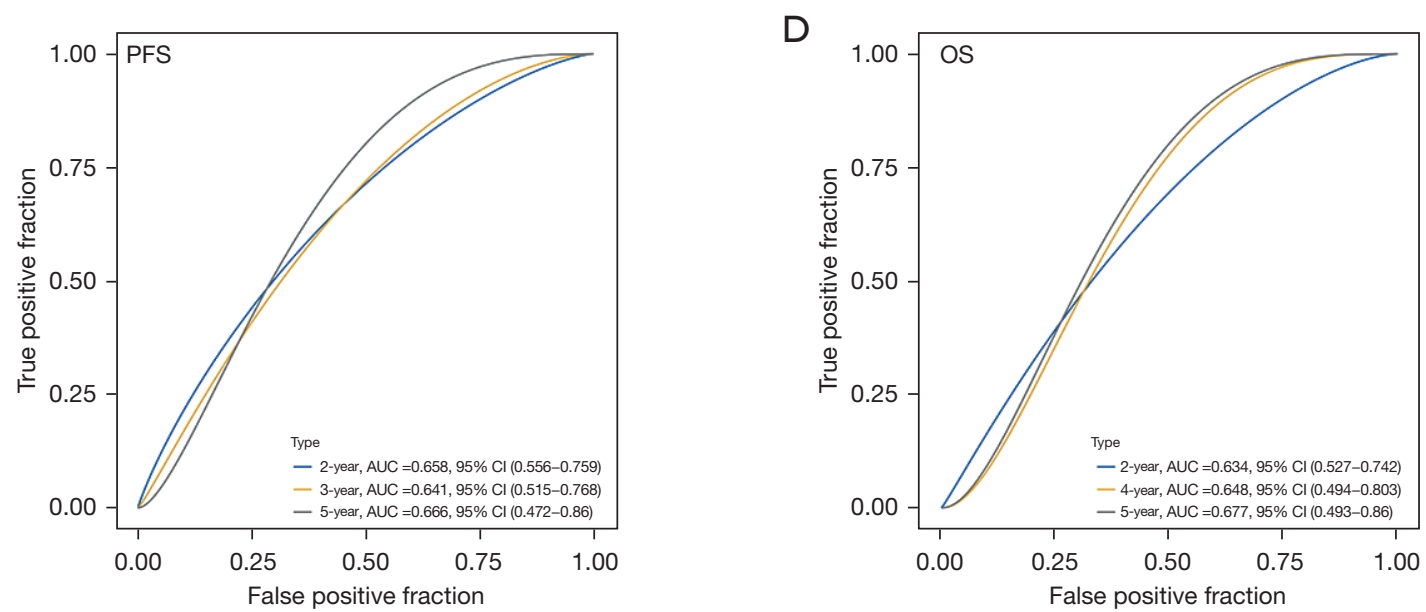

Figure 9 The prognostic value of CCR4 in HNSC patients with radiotherapy. (A) Kaplan-Meier survival analysis of PFS and CCR4 expression in HNSC patients with radiotherapy; (B) Kaplan-Meier survival analysis of OS and CCR4 expression in HNSC patients with radiotherapy; (C) ROC analysis of CCR4 on PFS at 2-, 3-, and 5-year follow-up in HNSC patients with radiotherapy; (D) ROC analysis of CCR4 on OS at 2-, 4-, and 5-year follow-up in HNSC patients with radiotherapy. CCR4, CC chemokine receptor 4; HNSC, head and neck squamous cell carcinoma. 
response, and the chemokine receptor CCR4 has mostly focused on it and T cells (33-35). CCR4 is predominantly expressed on the surface of regulatory $\mathrm{T}$ cells and helper Th2 cells, and mediates different types of biological effects in cells. Its related ligands include CCL17 and CCL22 (36). Adult peripheral blood data confirm that chemokines The receptor CCR4 can be detected on the surface of about $20 \%$ of effector T cells (37), and the number of Th2 cells is dominant. It has been confirmed in transplantation, inflammation and autoimmune diseases that Th2 cells are induced by CCR4 to bind to their ligands CCL17/CCL22 and then aggregate, and the polarization of Th2/Th1 cells is related to the development of their diseases (38).

Thymus and activation-regulated chemokine (TARC/ CCL17) and human macrophage-derived chemokine (hMDC/CCL22) produce different outcomes by binding with CCR4 $(39,40)$. CCL17 is expressed widely in more cell types, including both immune and nonimmune cells, compared with CCL22 which is limited to immune cells (41). CCL17 and CCL22 appear to compete to bind with CCR4. Treg cells and Th2 cells accumulate and infiltrate in the tumor microenvironment through the combination of CCL17/CCL22 and CCR4.

In some cases, CCR 4 acts as a tumor promoter. CCR 4 can recruit Tregs which can promote the immune escape of cancers (42). Tregs recruited by CCR4 have even been reported to evoke immunotherapy resistance (43). CCR4 is also believed to correlate with cancer metastasis. Previous studies have reported that CCR4 promoted lung metastasis in breast cancer, brain metastasis in melanoma, bone metastasis in lung cancer, and so on (44). However, other studies lean towards the opposite opinion $(19,45)$. Thus, the role of CCR4 in HNSC needs to be clarified.

In our study, we found the infiltrating level of many types of immune cells were positively related to the expression of CCR4, especially CD4+ T cells, CD8+ T cells, and B cells. High levels of CD8+ T cells and B cells predicted a better prognosis in HNSC in our study. Activated CD8+ T lymphocytes are thought to kill cancer cells by exerting antitumor effects and have been reported in many studies to indicate a good prognosis $(46,47)$. Other studies have also verified the good prognostic value of CD20+ B lymphocytes in cancers, and synergistic effects may exist between CD8+ T lymphocytes and B lymphocytes $(48,49)$. This might explain, to some extent, why patients with high expression of CCR4 had better prognoses in HNSC. Immune regulation is a complex process in vivo. Together with the existing evidence, the findings of our study showed that CCR4 played multifaceted roles in the immune microenvironment in different tumors. Further, we plotted the relationship between immune checkpoints and CCR4 to clarify the relationship between CCR 4 and tumor immune escape. These results confirmed that CCR4 also played an important role in the immune escape mechanism of tumors.

TP53 as a suppressor gene also plays an essential role in HNSC. In our study, we found that patients with a mutation of TP53 had a worse prognosis, which is consistent with the findings of the available literature. At the same time, we showed that the expression of CCR4 in wild-type TP53 significantly outyields that in mutant. In both the mutant TP53 and wild-type TP53 groups, high expression of CCR4 was a marker for a better prognosis.

To further investigate the functions of CCR 4 in HNSC, the transcriptome from TCGA was assessed by GSEA, which found that allograft rejection, inflammatory response, and IL-6 pathways were upregulated in the high CCR4 expression group. In the low CCR4 expression group, oxidative phosphorylation, DNA repair, MYC, reactive oxygen species pathway, and p53 pathway were enriched. Oxidative phosphorylation has been reported in many studies to promote tumor growth in vivo (50). MYC as an oncogene often regulates differentiation, proliferation, apoptosis, metabolism, and DNA repair of cancers (51). These are all possible mechanisms involved in prognosis.

Although radiotherapy is an important treatment method for HNSC patients, under simple radiotherapy irradiation, tumor cells often develop radiotherapy resistance, which is related to the repair of DNA damage of tumor cells after radiotherapy. After many years evaluating different prognostic markers of HNSC, we have still not found a validated biomarker to predict the response to radiotherapy. Therefore, we evaluated the prognostic value of CCR 4 in HNSC patients receiving radiotherapy. Our results indicated that CCR4 had a good prognosis in patients with radiotherapy. In short, CCR4 was a promising prognostic biomarker, but further research is needed to clarify this.

In summary, we suggested that CCR4 could be used as a prognostic biomarker for HNSC patients. At the same time, CCR 4 was also closely related to tumor immune infiltration, and it is hoped that it will become a new immunotherapy target. However, the specific mechanism of CCR4 overexpression in the development of HNSC is still unclear and further research is urgently needed.

Our study had several limitations. Different kinds of tumors have specific immune microenvironments and the mechanisms of these are still unclear. CCR4 may not be the 
driving force of a good prognosis in HNSC as we cannot ignore the synergistic effect of different immune cells. Nonetheless, in the unique immune microenvironment of HNSC, we did find that the high expression of CCR 4 correlated with a good prognosis. The second concern relates to the low sample number and missing treatment information, which might have introduced bias into our study; however, our results across different subgroups all proved our conclusion. Further studies with larger cohorts are necessary to confirm the results.

\section{Acknowledgments}

Funding: None.

\section{Footnote}

Reporting Checklist: The authors have completed the REMARK reporting checklist. Available at https://dx.doi. org/10.21037/atm-21-3936

Conflicts of Interest: All authors have completed the ICMJE uniform disclosure form (available at https://dx.doi. org/10.21037/atm-21-3936). The authors have no conflicts of interest to declare.

Ethical Statement: The authors are accountable for all aspects of the work in ensuring that questions related to the accuracy or integrity of any part of the work are appropriately investigated and resolved. The study was conducted in accordance with the Declaration of Helsinki (as revised in 2013).

Open Access Statement: This is an Open Access article distributed in accordance with the Creative Commons Attribution-NonCommercial-NoDerivs 4.0 International License (CC BY-NC-ND 4.0), which permits the noncommercial replication and distribution of the article with the strict proviso that no changes or edits are made and the original work is properly cited (including links to both the formal publication through the relevant DOI and the license). See: https://creativecommons.org/licenses/by-nc-nd/4.0/.

\section{References}

1. Travassos DC, Fernandes D, Massucato EMS, et al. Squamous cell carcinoma antigen as a prognostic marker and its correlation with clinicopathological features in head and neck squamous cell carcinoma: Systematic review and meta-analysis. J Oral Pathol Med 2018;47:3-10.

2. Sung H, Ferlay J, Siegel RL, et al. Global Cancer Statistics 2020: GLOBOCAN Estimates of Incidence and Mortality Worldwide for 36 Cancers in 185 Countries. CA Cancer J Clin 2021;71:209-49.

3. Castellsagué X, Alemany L, Quer M, et al. HPV Involvement in Head and Neck Cancers: Comprehensive Assessment of Biomarkers in 3680 Patients. J Natl Cancer Inst 2016;108:djv403.

4. Ang KK, Harris J, Wheeler R, et al. Human papillomavirus and survival of patients with oropharyngeal cancer. $\mathrm{N}$ Engl J Med 2010;363:24-35.

5. Sawant SS, Zingde SM, Vaidya MM. Cytokeratin fragments in the serum: their utility for the management of oral cancer. Oral Oncol 2008;44:722-32.

6. Wu XL, Tu Q, Faure G, et al. Diagnostic and Prognostic Value of Circulating Tumor Cells in Head and Neck Squamous Cell Carcinoma: a systematic review and metaanalysis. Sci Rep 2016;6:20210.

7. Kulasinghe A, Kenny L, Punyadeera C. Circulating tumour cell PD-L1 test for head and neck cancers. Oral Oncol 2017;75:6-7.

8. Röhrle N, Knott MML, Anz D. CCL22 Signaling in the Tumor Environment. Adv Exp Med Biol 2020;1231:79-96.

9. Yoshie O, Matsushima K. CCR4 and its ligands: from bench to bedside. Int Immunol 2015;27:11-20.

10. Mukherjee D, Zhao J. The Role of chemokine receptor CXCR4 in breast cancer metastasis. Am J Cancer Res 2013;3:46-57.

11. Weihrauch MR, Manzke O, Beyer M, et al. Elevated serum levels of CC thymus and activa-tion-related chemokine (TARC) in primary Hodgkin.s disease: Potential for a prognostic factor. Cancer Res 2005;65:5516-9.

12. Shimauchi T, Kabashima K, Tokura Y. Adult T-cell leukemia/lymphoma cells from blood and skin tumors express cytotoxic $T$ lymphocyte-associated antigen- 4 and Foxp3 but lack suppressor activity toward autologous CD8+ T cells. Cancer Sci 2008;99:98-106.

13. Lechner A,Schloser H, Rothschild SI, et al. Characterization of tumor-asociated T-lymphocyte subsets and immune checkpoint molecules in head and neck squamous cel carcinoma. Oncotarget 2017;8:44418-33.

14. Hu TH, Yao Y, Yu S, et al. SDF-1/CXCR4 promotes epithelial-mesenchymal transition and progression of colorectal cancer by activation of the $\mathrm{Wnt} / \beta$-catenin signaling pathway. Cancer Lett 2014;354:417-26.

15. Weide B, Allgaier N, Hector A, et al. Increased CCL17 
serum levels are associated with improved survival in advanced melanoma. Cancer Immunol Immunother 2015;64:1075-82.

16. Nakanishi T, Imaizumi K, Hasegawa Y, et al. Expression of macrophage-derived chemokine (MDC)/CCL22 in human lung cancer. Cancer Immunol Immunother 2006;5 5:1320-9.

17. Li T, Fan J, Wang B, et al. TIMER: A Web Server for Comprehensive Analysis of Tumor-Infiltrating Immune Cells. Cancer Res 2017;77:e108-10.

18. Summerfield A, McNeilly F, Walker I, et al. Depletion of CD4(+) and CD8(high+) T-cells before the onset of viraemia during classical swine fever. Vet Immunol Immunopathol 2001;78:3-19.

19. Meng L, He X, Hong Q, et al. CCR4, CCR8, and P2RY14 as Prognostic Factors in Head and Neck Squamous Cell Carcinoma Are Involved in the Remodeling of the Tumor Microenvironment. Front Oncol 2021;11:618187.

20. Imai T, Nagira $M$, Takagi S, et al. Selective recruitment of CCR4-bearing Th2 cells toward antigen-presenting cells by the CC chemokines thymus and activation-regulated chemokine and macrophage-derived chemokine. Int Immunol 1999;11:81-8.

21. Bonecchi R, Bianchi G, Bordignon PP, et al. Differential expression of chemokine receptors and chemotactic responsiveness of type $1 \mathrm{~T}$ helper cells (Th1s) and Th2s. J Exp Med 1998;187:129-34.

22. Sozzani S, Allavena P, Vecchi A, et al. The role of chemokines in the regulation of dendritic cell trafficking. J Leukoc Biol 1999;66:1-9.

23. Murphy PM, Baggiolini M, Charo IF, et al. International union of pharmacology. XXII. Nomenclature for chemokine receptors. Pharmacol Rev 2000;52:145-76.

24. Cowan JE, McCarthy NI, Parnell SM, et al. Differential requirement for CCR4 and CCR7 during the development of innate and adaptive $\alpha \beta T$ cells in the adult thymus. J Immunol 2014;193:1204-12.

25. Mishalian I, Bayuh R, Eruslanov E, et al. Neutrophils recruit regulatory $\mathrm{T}$-cells into tumors via secretion of CCL17--a new mechanism of impaired antitumor immunity. Int J Cancer 2014;135:1178-86.

26. Gniadecki R. CCR4-targeted therapy in cutaneous T-cell lymphoma. Lancet Oncol 2018;19:1140-1.

27. Li JY, Ou ZL, Yu SJ, et al. The chemokine receptor CCR4 promotes tumor growth and lung metastasis in breast cancer. Breast Cancer Res Treat 2012;131:837-48.

28. Zhao H, Bo Q, Wang W, et al. CCL17-CCR4 axis promotes metastasis via ERK/MMP13 pathway in bladder cancer. J Cell Biochem 2018. [Epub ahead of print]. doi: 10.1002/jcb.27494.

29. Sohn EJ, Jung DB, Lee H, et al. CNOT2 promotes proliferation and angiogenesis via VEGF signaling in MDA-MB-231 breast cancer cells. Cancer Lett 2018;412:88-98.

30. Godiska R, Chantry D, Raport CJ, et al. Human macrophage-derived chemokine (MDC), a novel chemoattractant for monocytes, monocyte-derived dendritic cells, and natural killer cells. J Exp Med 1997;185:1595-604.

31. Tan MC, Goedegebuure PS, Belt BA, et al. Disruption of CCR5-dependent homing of regulatory $T$ cells inhibits tumor growth in a murine model of pancreatic cancer [J]. J Immunol 2009;182:1746-55.

32. Wang Y, Zhang Y, Yang X, et al. Chemokine-like factor 1 is a functional ligand for CC chemokine receptor 4 (CCR4). Life Sci 2006;78:614-21.

33. Ghadjar P, Rubie C, Aebersold DM, et al. The chemokine CCL20 and its receptor CCR6 in human malignancy with focus on colorectal cancer. Int J Cancer 2009;125:741-5.

34. Hu D, Du C, et al. The expression of chemokine receptors CCR6, CXCR2 and CXCR4 is not organ-specific for distant metastasis in colorectal cancer: a comparative study. Histopathology 2013;63:167-73.

35. Rubie C, Frick VO, et al. CCL20/CCR6 expression profile in pancreatic cancer. J Transl Med 2010;8:45.

36. Ishida T, Ueda R. CCR 4 as a novel molecular target for immunotherapy of cancer. Cancer Sci 2006;97:1139-46.

37. Ohshima K, Karube K, et al. Classification of distinct subtypes of peripheral T-cell lymphoma unspecified, identified by chemokine and chemokine receptor expression: Analysis of prognosis. Int J Oncol 2004;25:605-13.

38. Nicolls MR, Coulombe $M$, et al. Interferon-gamma is not a universal requirement for islet allograft survival. Transplantation 2002;74:472-7.

39. Santulli-Marotto S, Boakye K, Lacy E, et al. Engagement of two distinct binding domains on CCL17 is required for signaling through CCR4 and establishment of localized inflammatory conditions in the lung. PLoS One 2013;8:e81465.

40. Viney JM, Andrew DP, Phillips RM, et al. Distinct conformations of the chemokine receptor CCR4 with implications for its targeting in allergy. J Immunol 2014;192:3419-27.

41. Gobert M, Treilleux I, Bendriss-Vermare N, et al. Regulatory $\mathrm{T}$ cells recruited through CCL22/CCR4 are 
selectively activated in lymphoid infiltrates surrounding primary breast tumors and lead to an adverse clinical outcome. Cancer Res 2009;69:2000-9.

42. Marshall LA, Marubayashi S, Jorapur A, et al. Tumors establish resistance to immunotherapy by regulating Treg recruitment via CCR4. J Immunother Cancer 2020;8:e000764.

43. Nakamura ES, Koizumi K, Kobayashi M, et al. RANKLinduced CCL22/macrophage-derived chemokine produced from osteoclasts potentially promotes the bone metastasis of lung cancer expressing its receptor CCR4. Clin Exp Metastasis 2006;23:9-18.

44. Klein A, Sagi-Assif O, Meshel T, et al. CCR4 is a determinant of melanoma brain metastasis. Oncotarget 2017;8:31079-91.

45. Zhang Z, Liu C, Liang T, et al. Establishment of immune prognostic signature and analysis of prospective molecular mechanisms in childhood osteosarcoma patients. Medicine (Baltimore) 2020;99:e23251.

46. Vihervuori H, Autere TA, Repo H, et al. Tumorinfiltrating lymphocytes and CD8+ T cells predict survival

Cite this article as: Zhang $\mathrm{Y}$, Chen K, Li L, Mao W, Shen D, Yao N, Zhang L. CCR4 is a prognostic biomarker and correlated with immune infiltrates in head and neck squamous cell carcinoma. Ann Transl Med 2021;9(18):1443. doi: 10.21037/atm-21-3936 of triple-negative breast cancer. J Cancer Res Clin Oncol 2019;145:3105-14.

47. E J, Yan F, Kang Z, et al. CD8+CXCR5+ T cells in tumordraining lymph nodes are highly activated and predict better prognosis in colorectal cancer. Hum Immunol 2018;79:446-52.

48. Edin S, Kaprio T, Hagström J, et al. The Prognostic Importance of CD20+ B lymphocytes in Colorectal Cancer and the Relation to Other Immune Cell subsets. Sci Rep 2019;9:19997.

49. Sakimura C, Tanaka H, Okuno T, et al. B cells in tertiary lymphoid structures are associated with favorable prognosis in gastric cancer. J Surg Res 2017;215:74-82.

50. Sica V, Bravo-San Pedro JM, Stoll G, et al. Oxidative phosphorylation as a potential therapeutic target for cancer therapy. Int J Cancer 2020;146:10-7.

51. Duffy MJ, O'Grady S, Tang M, et al. MYC as a target for cancer treatment. Cancer Treat Rev 2021;94:102154.

(English Language Editor: A. Muijlwijk) 
Table S1 Characteristics of the Cancer Genome Atlas (TCGA)head and neck squamous cell carcinoma (HNSC) patients

\begin{tabular}{|c|c|}
\hline Characteristic & Overall $(n=502)$ \\
\hline \multicolumn{2}{|l|}{ T stage, $\mathrm{n}(\%)$} \\
\hline $\mathrm{T} 1$ & $33(6.8)$ \\
\hline T2 & $144(29.6)$ \\
\hline T3 & $131(26.9)$ \\
\hline T4 & $179(36.8)$ \\
\hline \multicolumn{2}{|l|}{ N stage, n (\%) } \\
\hline No & $239(49.8)$ \\
\hline N1 & $80(16.7)$ \\
\hline N2 & $154(32.1)$ \\
\hline N3 & $7(1.5)$ \\
\hline \multicolumn{2}{|l|}{ M stage, n (\%) } \\
\hline Mo & 472 (99.0) \\
\hline M1 & $5(1.0)$ \\
\hline \multicolumn{2}{|c|}{ Clinical stage, n (\%) } \\
\hline Stage I & $19(3.9)$ \\
\hline Stage II & $95(19.5)$ \\
\hline Stage III & $102(20.9)$ \\
\hline Stage IV & $272(55.7)$ \\
\hline \multicolumn{2}{|c|}{ Radiation therapy, n (\%) } \\
\hline No & 154 (34.9) \\
\hline Yes & $287(65.1)$ \\
\hline \multicolumn{2}{|l|}{ Gender, n (\%) } \\
\hline Female & $134(26.7)$ \\
\hline Male & 368 (73.3) \\
\hline \multicolumn{2}{|l|}{ Age, n (\%) } \\
\hline$\leq 60$ & 245 (48.9) \\
\hline$>60$ & $256(51.1)$ \\
\hline \multicolumn{2}{|c|}{ Histologic grade, n (\%) } \\
\hline G1 & $62(12.8)$ \\
\hline G2 & $300(62.1)$ \\
\hline G3 & $119(24.6)$ \\
\hline G4 & $2(0.4)$ \\
\hline \multicolumn{2}{|l|}{ Smoker, n (\%) } \\
\hline No & $111(22.6)$ \\
\hline Yes & $381(77.4)$ \\
\hline \multicolumn{2}{|c|}{ Alcohol history, n (\%) } \\
\hline No & $158(32.2)$ \\
\hline Yes & $333(67.8)$ \\
\hline
\end{tabular}

